\title{
The Diagnostic Value of CT Myelography, MR Myelography, and Both in Neonatal Brachial Plexus Palsy
}

\author{
R. Tse, J.N. Nixon, R.S. Iyer, K.A. Kuhlman-Wood, and G.E. Ishak
}

\begin{abstract}
BACKGROUND AND PURPOSE: Although most infants with brachial plexus palsy recover function spontaneously, approximately 10 -30\% benefit from surgical treatment. Pre-operative screening for nerve root avulsions is helpful in planning reconstruction. Our aim was to compare the diagnostic value of CT myelography, MR myelography, and both against a surgical criterion standard for detection of complete nerve root avulsions in birth brachial plexus palsy.
\end{abstract}

MATERIALS AND METHODS: Nineteen patients who underwent a preoperative CT and/or MR myelography and subsequent brachial plexus exploration were included. Imaging studies were analyzed for the presence of abnormalities potentially predictive of nerve root avulsion. Findings of nerve root avulsion on surgical exploration were used as the criterion standard to assess the predictive value of imaging findings.

RESULTS: Ninety-five root levels were examined. When the presence of any pseudomeningocele was used as a predictor, the sensitivity was 0.73 for CT and 0.68 for MR imaging and the specificity was 0.96 for $C T$ and 0.97 for MR imaging. When presence of pseudomeningocele with absent rootlets was used as the predictor, the sensitivity was 0.68 for CT and 0.68 for MR imaging and the specificity was 0.96 for CT and 0.97 for MR imaging. The use of both CT and MR imaging did not increase diagnostic accuracy. Rootlet findings in the absence of pseudomeningocele were not helpful in predicting complete nerve root avulsion.

CONCLUSIONS: Findings of CT and MR myelography were highly correlated. Given the advantages of MR myelography, it is now the single technique for preoperative evaluation of nerve root avulsion at our institution.

B rachial plexus palsy occurs in approximately 1 in 1000 neonates. ${ }^{1,2}$ Downward traction on the shoulder girdle produces stereotyped patterns of plexus injury. ${ }^{3}$ Nerve lesions occur first at higher levels, with more severe traction resulting in progressive inferior extension. ${ }^{3,4}$ More superior nerve injury is typically extraforaminal, at the level of the superior trunks, because a welldeveloped investing fascia protects the upper nerve roots from proximal traction. In contrast, inferior lesions are more often intraforaminal, manifesting as either partial or complete avulsion of the nerve root. ${ }^{4}$

Clinical manifestations and spontaneous recovery depend on the extent, location, and type of nerve lesions. The clinical presen-

Received October 17, 2013; accepted after revision December 16.

From the Department of Radiology (J.N.N., R.S.L., G.E.I.), and the Division of Plastic Surgery, Department of Surgery (R.T., K.A.K.-W.), the Seattle Children's Hospital, University of Washington, Seattle, Washington.

Paper previously presented at: XVIII International Symposium on Brachial Plexus Surgery, May 23-25, 2013; Montreux, Switzerland.

Please address correspondence to Raymond Tse, M/S OB.9.527, 4800 Sand Point Way NE, Seattle, WA 98105; e-mail: raymond.tse@seattlechildrens.org

http://dx.doi.org/10.3174/ajnr.A3878 tation can generally be grouped into 1 of 4 patterns outlined by Narakas ${ }^{5}$ : Type I involves C5 and C6 deficits (Erb-Duchenne type) with loss of shoulder abduction, shoulder external rotation, elbow flexion, and forearm supination. Type II involves C5 to C7/C8 deficits, resulting in a "waiter's tip" posture from additional loss of wrist extension. Type III involves C5 to C8/T1 deficits, resulting in an arm that is generally paralyzed. Type IV involves $\mathrm{C} 5$ to $\mathrm{T} 1$ and the sympathetic chain, resulting in a flail arm with Horner syndrome. Upward traction on the brachial plexus can result in isolated lower plexus deficits that manifest as paralysis of the hand only. ${ }^{6,7}$ This pattern is known as Klumpke palsy.

The decision to proceed with surgical exploration and reconstruction is based on the clinical presentation and progression. While $70 \%-90 \%$ of infants are treated with therapy alone, $10 \%-$ $30 \%$ have indications for surgical treatment. ${ }^{8-11}$ Nerve injuries distal to the intervertebral foramen can be reconstructed by using nerve grafts, whereas intraforaminal nerve root avulsions require nerve transfer. While both partial and complete nerve root avulsions are described, ${ }^{12,13}$ there is no clear consensus on the surgical approach to partial nerve root avulsions. Preoperative imaging capable of accurately identifying complete nerve root avulsions 
and distinguishing them from extraforaminal nerve injuries is, therefore, critical for optimal surgical planning.

The current standard for preoperative assessment of nerve root avulsions in infants is CT myelography. ${ }^{12,14-19}$ A pseudomeningocele is suggestive of nerve root avulsion, and the additional finding of absent rootlets traversing the pseudomeningocele greatly increases the specificity of this finding. ${ }^{14} \mathrm{CT}$ myelography requires a lumbar puncture for injection of intrathecal contrast, with attendant risks of infection and seizure. ${ }^{20-22}$ Recent studies have also raised concern for malignancy with early exposure of children to radiation. ${ }^{23,24}$ MR myelography can be performed without injection of contrast and is a promising alternative. ${ }^{17,25}$ However, the performance of MR myelography for predicting nerve root avulsion is not yet established ${ }^{26}$ in neonatal brachial plexus injury, and the diagnostic value of MR myelography has yet to be compared with CT myelography in this setting.

The purpose of this study was to determine the predictive value of CT myelography, MR myelography, and both CT and MR myelography for detecting complete nerve root avulsions in neonatal brachial plexus palsy, by using a surgical criterion standard.

\section{MATERIALS AND METHODS}

This study was approved by the institutional review board and was conducted in compliance with Health Insurance Portability and Accountability Act guidelines. Informed consent for participation was waived, given that evaluation was retrospective and data were pre-existing.

\section{Subjects}

All consecutive patients with neonatal brachial plexus palsy who underwent surgical exploration at our institution (from November 2009 to May 2013) and who had preoperative CT and/or MR myelography were included in this study. Indications for surgical treatment followed the protocol developed at the Toronto Hospital for Sick Children ${ }^{9}$ and were based on clinical examination: flail arm and persistent Horner syndrome at 1 month of age; composite active movement scale score for elbow flexion, elbow extension, wrist extension, finger extensions, and thumb extension of $<3.5 / 10$ at 3 months of age; no clinical progression at 6 months of age; and failed "cookie test" (the child has to bring the hand to the mouth) at 9 months of age. One infant with isolated lower plexus palsy underwent exploration at 9 months of age because of lack of clinical recovery. Myelography was performed only on infants in whom a clinical decision to proceed with surgical exploration was made. Subject demographics were collected by retrospective chart review.

\section{Imaging Studies}

CT myelography was performed with a 64-detector LightSpeed CT scanner (GE Healthcare, Milwaukee, Wisconsin) following intrathecal injection of iopamidol iodinated contrast material (Isovue-M 200; General Injectables and Vaccines, Bastian, Virginia) under fluoroscopic guidance according to a weight-based protocol of $0.5 \mathrm{~mL} / \mathrm{kg}$ with a maximum dose of $5 \mathrm{~mL}$. Axial $0.625-\mathrm{mm}$ sections were reconstructed from a volumetric acquisition extending from the skull base to T4 by using both standard and
Table 1: Potential findings on myelography

\begin{tabular}{cll} 
& \multicolumn{2}{c}{ Finding } \\
\cline { 2 - 3 } Rating & \multicolumn{1}{c}{ Dura } & Rootlets \\
\hline A & Pseudomeningocele & Absent \\
B & Pseudomeningocele & Present \\
C & Normal & Absent \\
D & Normal & Thinned \\
E & Normal & Thickened \\
F & Normal & Normal \\
\hline
\end{tabular}

sharpening convolution kernels. A pitch of $0.53: 1$, reconstruction increment of $0.4 \mathrm{~mm}$, beam width of $20 \mathrm{~cm}$, focal spot size of $0.6 \times 0.7 \mathrm{~mm}$, matrix size of $512 \times 512$, and an FOV of $10 \mathrm{~cm}$ were used. Data were reconstructed into sagittal and curved coronal planes for optimal nerve root assessment. Kilovolt(peak) and milliampere values of 100 and 155 were used.

MR imaging examinations were performed on a 3T TrioTim MR imaging (Siemens, Erlangen, Germany) following a MR myelography protocol. Sequences included coronal and sagittal STIR, coronal and sagittal T1-weighted, and a fully-rewound coherent steady-state gradient-echo sequence with dual excitation (constructive interference in steady state on the Siemens platform) acquired at high resolution. Resolution of the steady-state sequence varied between 0.5 and $0.9 \mathrm{~mm}$ isotropic, and the time of the acquisition varied between 2 minutes 11 seconds and 7 minutes 38 seconds depending on plane, resolution, and coverage.

None of the imaging studies were excluded on the basis of study quality, so our results represented true clinical practice.

\section{Blinded Myelogram Findings}

CT and MR myelograms were de-identified, unlinked, randomized, and loaded onto a test PACS system. A subject key code was stored securely, and the participating radiologists were blinded to the identity of each scan and the results of surgical exploration. The side of the clinical deficit was provided, and the contralateral side was used for comparison.

Two pediatric radiologists (with 7 and 8 years' experience, respectively) independently evaluated each imaging study and rated each root level from $\mathrm{C} 5$ to $\mathrm{T} 1$ according to the system in Table 1. Discrepancies were resolved by consensus analysis. Findings $A$ and $B$ (Figs 1 and 2) have previously been used as predictors of nerve root avulsion in infants. ${ }^{14}$ Finding $\mathrm{C}$ (Fig 3) has been described as a predictor in adults. ${ }^{19}$ Findings $\mathrm{D}$ and $\mathrm{E}$ (Figs 4 and 5) have been suggested to indicate partial nerve root avulsion. ${ }^{12}$

To determine the subjective quality of each type of myelogram, each radiologist rated their confidence in their findings at each root level by using a 3-point scale: 1 , absolutely sure; 2, likely; 3 , unsure.

\section{Surgical Findings}

Brachial plexus exploration involved a supraclavicular approach with retroclavicular and infraclavicular exposures as needed. Each nerve root was dissected proximal to the intervertebral foramen for inspection. A nerve root was considered completely avulsed when we found any of the following: 

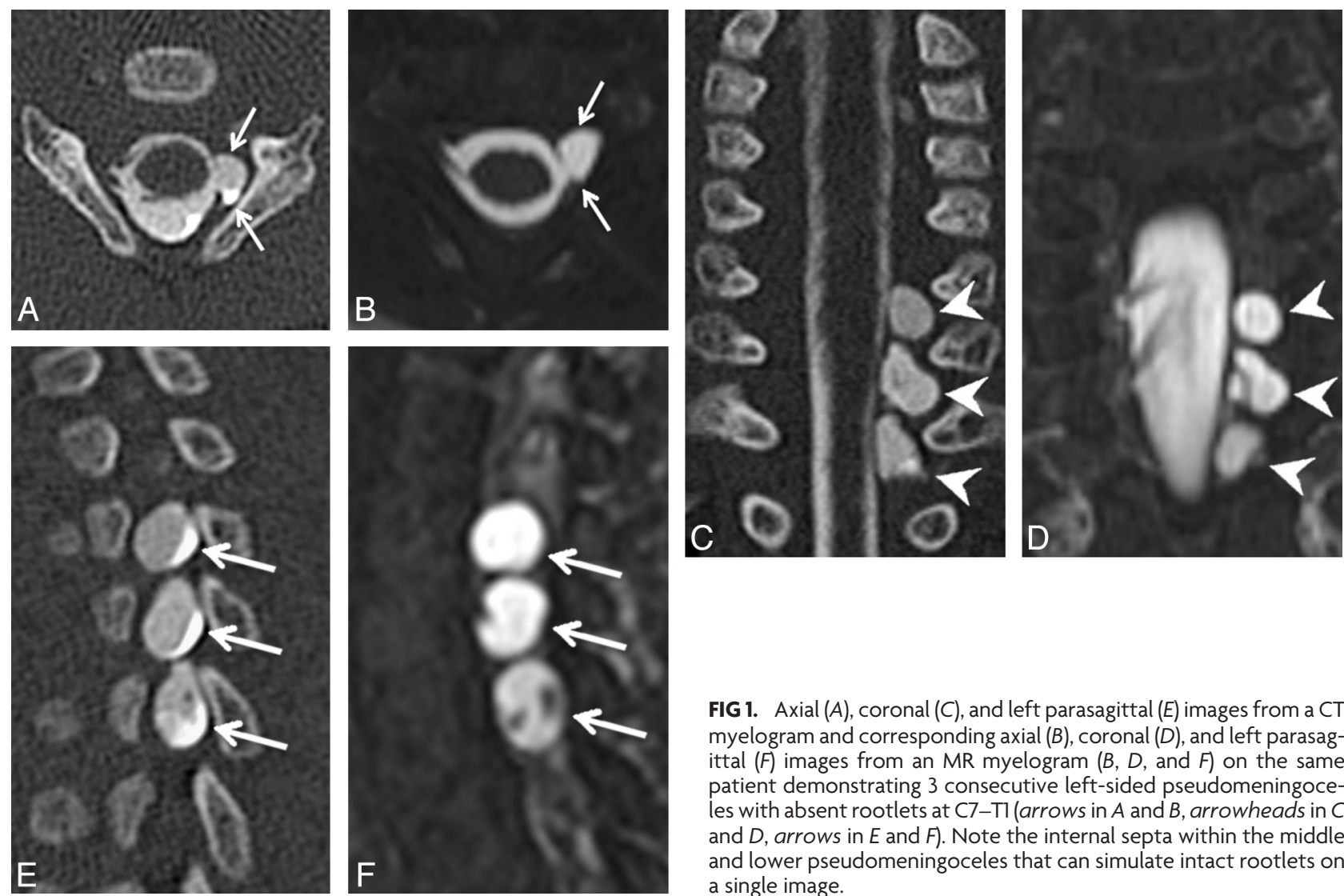

FIG 1. Axial $(A)$, coronal $(C)$, and left parasagittal $(E)$ images from a $C T$ myelogram and corresponding axial $(B)$, coronal $(D)$, and left parasagittal $(F)$ images from an MR myelogram $(B, D$, and $F)$ on the same patient demonstrating 3 consecutive left-sided pseudomeningoceles with absent rootlets at C7-T1 (arrows in $A$ and $B$, arrowheads in $C$ and $D$, arrows in $E$ and $F$. Note the internal septa within the middle and lower pseudomeningoceles that can simulate intact rootlets on a single image.

3) There was a normal-appearing nerve with no response to

1) The dorsal root ganglion was identified outside the intervertebral foramen.

2) The intervertebral foramen was empty. electrical stimulation on exploration, no clinical function on preoperative examination, and no distal lesion identified.

We did not define partial nerve root
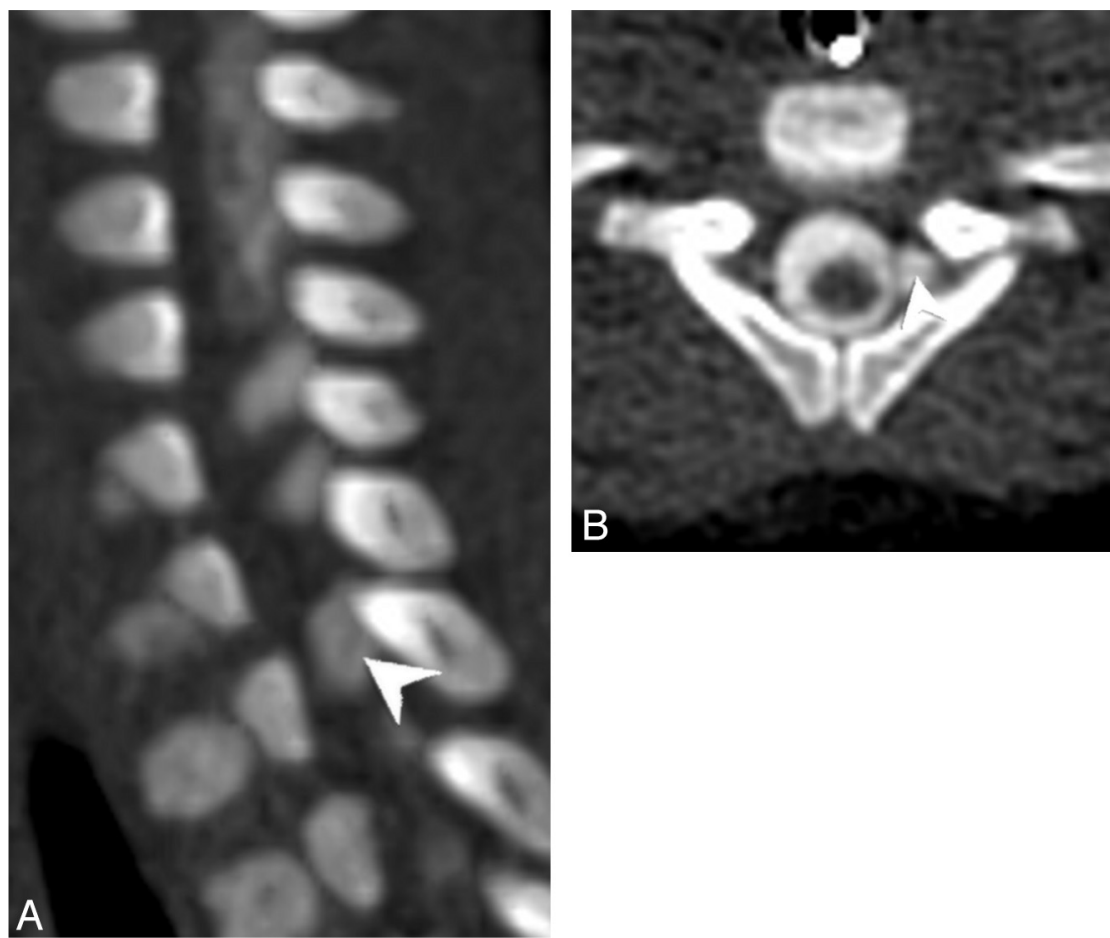
avulsion based on surgical findings, given that this would require laminectomy for intraspinal exploration, and this is not performed for neonatal brachial plexus palsy.

\section{Statistical Analysis}

The radiologic findings on preoperative myelograms were compared with the surgical findings. The operative findings were considered the criterion standard. The diagnostic accuracy of each of the predictors identified on myelography was analyzed, and the sensitivity, specificity, positive predictive value, negative predictive value, and likelihood ratio for complete root avulsion of each were calculated.

Confidence ratings by each radiologist for each root level were compared for CT and MR myelography with a $\chi^{2}$ test by using STATA (StataCorp, College Station, Texas). In contrast to other studies, which excluded imaging studies based on poor quality or technical error, ${ }^{12,14}$ we included all

FIG 2. Left parasagittal $(A)$ and axial $(B)$ images from a CT myelogram demonstrating a left-sided pseudomeningocele at TI. A thin linear filling defect within the pseudomeningocele continued centrally to the cord and was interpreted as an intact nerve root. 


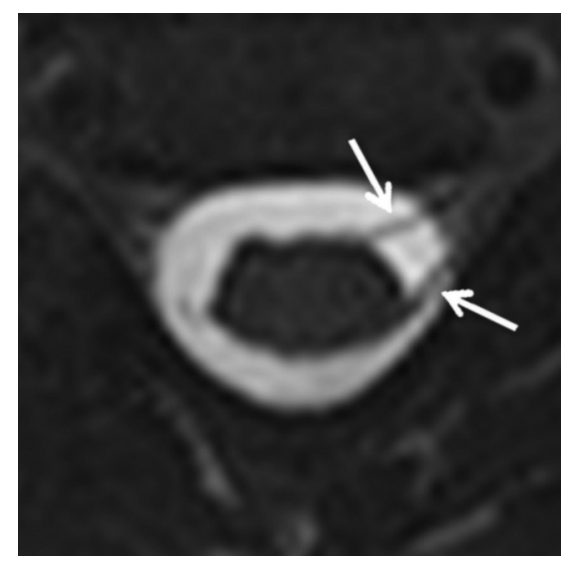

FIG 3. Axial minimum-intensity-projection image from an MR myelogram demonstrating only left-sided rootlets at C5 (arrows), with absent corresponding right-sided rootlets. The base resolution was $0.6-\mathrm{mm}$ isotropic, with a $2-\mathrm{mm}$ minimum-intensity-projection slab.

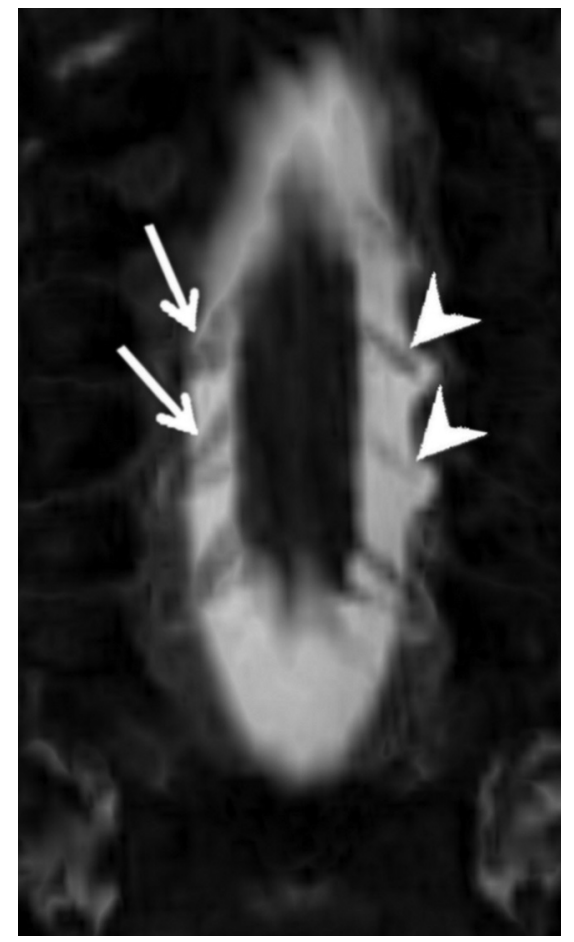

FIG 4. Coronal minimum-intensity-projection image from an MR myelogram demonstrating thinned left-sided ventral rootlets at $\mathrm{C} 6$ and C7 (arrowheads), relative to the normal fan-shaped right-sided ventral rootlets on the contralateral side at the same levels (arrows). Base resolution was $0.6-\mathrm{mm}$ isotropic, with a $2-\mathrm{mm}$ minimum-intensityprojection slab.

imaging studies to allow us to compare the 2 modalities in a practical clinical setting.

\section{RESULTS}

\section{Subjects and Surgical Findings}

During a 3.5-year period (between November 2009 and May 2013), 226 children presented to the Brachial Plexus Program at our institution. Of these new visits, 116 children were younger than 18 months of age at presentation. Nineteen of the 116 infants (16\%) underwent surgical exploration, and all met the inclusion criteria for this study. Seventeen patients had both CT and MR myelography performed preoperatively. Two subjects underwent CT myelography alone without concomitant MR myelography. All imaging studies were included in this study.

The male/female ratio was 9:10. The mean gestational age was 39.5 weeks (range, 36-41 weeks), and the mean birth weight was $3978 \mathrm{~g}$ (range, 2580-4479 g). Seventeen infants (89\%) presented in the cephalic position at vaginal delivery, and none presented breech. Two infants (11\%) were born by cesarean delivery. Brachial plexus palsies were identified immediately after birth. Two infants had ischemic encephalopathy, 1 had a clavicle fracture, 2 had humerus fractures, and 6 had torticollis. Infants were followed clinically, and myelography was only performed if a decision was made to proceed with surgical exploration. The overall mean age at myelography was 25 weeks (range, 10-65 weeks). Incidence, age at myelography, and avulsions on surgical exploration according to clinical presentation are summarized in Table 2. Ten subjects (52\%) had nerve root avulsions. There was an increasing incidence of root avulsion with increasing severity of injury according to the Narakas classification. Avulsions occurred more often in the lower roots.

Ninety-five root levels were examined (5 ipsilateral levels in 19 patients). There were no abnormalities detected on contralateral levels. Twenty-two avulsions were identified on surgical exploration, giving an overall incidence of $23 \%$. The distribution of avulsions according to root level and palsy type is summarized in Table 2.

\section{Predictive Value of Findings on Myelography}

Table 3 summarizes the predictive values of CT myelography, MR myelography, and both CT and MR myelography for all root levels by using either pseudomeningocele with absent rootlets or all pseudomeningoceles as indicators of complete nerve root avulsion. No benefit of CT and MR myelography combined was found. The findings and predictive values of CT myelography compared with MR myelography were almost the same and were consistent with those previously reported in the literature. ${ }^{12,14-16,18,19}$

Other nerve root findings in the absence of pseudomeningocele were also analyzed (absent rootlets, thinned rootlets, and thickened rootlets). These findings did not improve the predictive values for CT, MR imaging, or both CT and MR myelography (Table 4). We found no association between the presence or type of additional findings and age at imaging.

\section{Predictive Value According to Root Level}

The predictive values of CT myelography alone, MR myelography alone, and both $\mathrm{CT}$ and MR myelography could not be determined according to root level by using quantitative methods, given the limited cohort size. For all clinical presentations, there were no avulsions of $\mathrm{C} 5$ and there were only 3 avulsions of C6 in 19 subjects (Table 5). Qualitative analysis revealed little variation in the predictive value according to root level.

\section{Confidence Ratings}

Confidence ratings (190 scores for CT and 170 scores for MR imaging) were pooled according to imaging technique. There was 

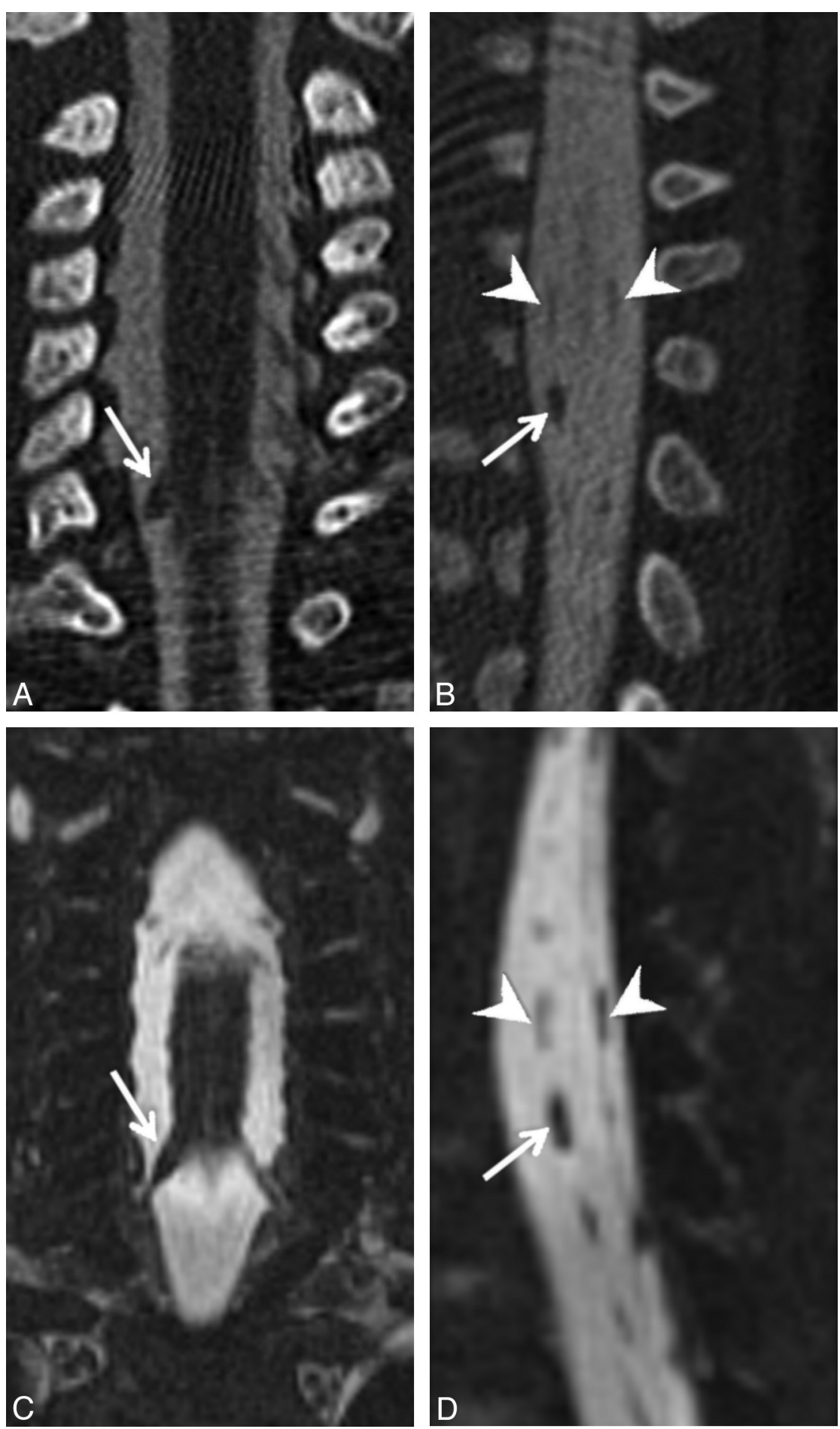

FIG 5. Coronal ( $A$ ) and right parasagittal ( $B$ ) images from a $C T$ myelogram and the coronal $(C)$ and right parasagittal $(D)$ images from the corresponding MR myelogram demonstrating a thickened ventral rootlet at $\mathrm{C} 8$ on the right (arrows in $A$ through $D$ ). Note that the dorsal rootlet at $\mathrm{C} 8$ is thinned. Normal caliber ventral and dorsal rootlets at C7 (arrowheads in $B$ and $D$ ) are visible for comparison.

significantly better confidence on CT myelography compared with MR myelography $(P<.01)$.

\section{DISCUSSION}

Preoperative assessment of nerve root avulsion is useful for surgical planning for brachial plexus palsy. Although CT myelography is the established standard in adults ${ }^{13,18,19,26-28}$ and in- fants, ${ }^{12,14,15,29}$ the risk of infection and seizure related to intrathecal contrast administration $^{20-22}$ and evidence that early exposure to radiation may increase later risks of malignancy ${ }^{23,24}$ make identifying an alternative important.

MR myelography for brachial plexus palsy has evolved during the past decade. ${ }^{30,31}$ Its predictive value for detecting nerve root avulsions has been evalu$\operatorname{ated}^{32-35}$ and has been found to have similar $^{36,37}$ or greater ${ }^{38}$ value compared with CT myelography in adults. MR myelography has also been evaluated in neonatal brachial plexus palsy ${ }^{25,39-44}$ but is yet to be widely adopted. Medina et $\mathrm{al}^{43}$ demonstrated good sensitivity and specificity for the detection of extraforaminal neuromas by using an MR imaging-based technique, but sensitivity for the detection of findings reflecting proximal nerve root avulsions, particularly characterization of the nerve roots themselves, was poor. In addition, the predictive value of MR imaging for complete nerve root avulsion is yet to be compared in a side-by-side manner with $\mathrm{CT}$, the current standard in infants.

Relative to previous studies, we used newer MR imaging technology. In our study, MR myelography was equal to and perhaps better than CT myelography for the prediction of complete nerve root avulsions on surgical exploration, and we found no benefit to the combined use of CT and MR imaging over MR myelography alone. This outcome supports the findings of several prior studies that evaluated the diagnostic performance of MR myelography alone $e^{25,39-44}$ and is further evidence that with the current technique, MR myelography may be capable of replacing CT myelography in the preoperative assessment of infants with neonatal brachial plexus palsy. In addition, MR imaging has the advantage of evaluating the intrinsic signal intensity and integrity of the spinal cord in better detail compared with CT. Increased use of MR myelography will potentially allow a decrease in radiation exposure and morbidity associated with invasive myelography.

We evaluated specific predictors of nerve root avulsion (pseudomeningocele with or without visible rootlets) on both $\mathrm{CT}$ and MR imaging and found that the predictive value of these findings was similar to that in other published studies. ${ }^{14}$ 
Table 2: Subject demographics

\begin{tabular}{|c|c|c|c|c|c|c|c|c|}
\hline \multirow[b]{2}{*}{ Clinical Presentation } & \multirow{2}{*}{$\begin{array}{l}\text { Subjects } \\
\text { (No.) }\end{array}$} & \multirow{2}{*}{$\begin{array}{l}\text { Mean Age at } \\
\text { Myelography } \\
\text { (wk) (Range) }\end{array}$} & \multicolumn{5}{|c|}{$\begin{array}{l}\text { Nerve Root Avulsion on } \\
\text { Surgical Exploration }\end{array}$} & \multirow{2}{*}{$\begin{array}{l}\text { Mean Avulsions per } \\
\text { Subject (All Levels) }\end{array}$} \\
\hline & & & C5 & $\mathrm{C6}$ & $\mathrm{C7}$ & $\mathrm{C} 8$ & $\mathrm{T1}$ & \\
\hline Narakas I & $4(21 \%)$ & $52(46-65)$ & $0 \%$ & $0 \%$ & $0 \%$ & $0 \%$ & $0 \%$ & 0 \\
\hline Narakas II & $4(21 \%)$ & $32(16-49)$ & $0 \%$ & $25 \%$ & $0 \%$ & $0 \%$ & $0 \%$ & 0.25 \\
\hline Narakas III & $3(16 \%)$ & 20 (14-27) & $0 \%$ & $33 \%$ & $66 \%$ & $33 \%$ & $66 \%$ & 2 \\
\hline Narakas IV & $7(37 \%)$ & $14(10-16)$ & $0 \%$ & $14 \%$ & $57 \%$ & $71 \%$ & $71 \%$ & 2.14 \\
\hline Klumpke & $1(5 \%)$ & 14 (NA) & $0 \%$ & $0 \%$ & $0 \%$ & $0 \%$ & $0 \%$ & 0 \\
\hline
\end{tabular}

Note:-NA indicates not applicable.

Table 3: Predictive value of $C T$ versus MRI versus $C T$ and MR myelography

\begin{tabular}{|c|c|c|c|c|c|c|}
\hline & \multicolumn{3}{|c|}{ Pseudomeningoceles with Absent Rootlets } & \multicolumn{3}{|c|}{ All Pseudomeningoceles } \\
\hline & CT & MRI & CT and MRI & CT & MRI & CT and MRI \\
\hline Sensitivity & 0.68 & 0.68 & 0.68 & 0.73 & 0.68 & 0.68 \\
\hline Specificity & 0.96 & 0.97 & 0.97 & 0.96 & 0.97 & 0.97 \\
\hline Positive predictive value & 0.83 & 0.88 & 0.88 & 0.84 & 0.88 & 0.88 \\
\hline Negative predictive value & 0.9 & 0.9 & 0.9 & 0.92 & 0.9 & 0.9 \\
\hline Likelihood ratio & 17 & 22.7 & 22.7 & 18 & 22.7 & 22.7 \\
\hline
\end{tabular}

Table 4: Likelihood ratios of detecting nerve root avulsions using different imaging predictors

\begin{tabular}{|c|c|c|c|c|}
\hline \multirow[b]{2}{*}{ Predictors of Nerve Root Avulsion } & \multirow{2}{*}{$\begin{array}{l}\text { Findings on } \\
\text { Imaging }\end{array}$} & \multicolumn{3}{|c|}{ Likelihood Ratio } \\
\hline & & CT & MRI & CT and MRI \\
\hline Pseudomeningoceles with absent rootlets & A & 17 & 22.7 & 22.7 \\
\hline Any pseudomeningocele & $A$ and $B$ & 18 & 22.7 & 22.7 \\
\hline Any pseudomeningocele or any absent rootlets & $\mathrm{A}, \mathrm{B}$, and $\mathrm{C}$ & 18 & 13.6 & 13.6 \\
\hline Any pseudomeningocele or any rootlet abnormality & $A, B, C, D$, and $E$ & 3.7 & 5.4 & 5.4 \\
\hline
\end{tabular}

Table 5: Avulsions on surgical exploration according to root level

\begin{tabular}{lcc}
\hline & \multicolumn{2}{c}{ Findings on Surgical Exploration } \\
\cline { 2 - 3 } $\begin{array}{c}\text { Root } \\
\text { Level }\end{array}$ & $\begin{array}{c}\text { Avulsions } \\
\text { (Roots Examined) }\end{array}$ & $\begin{array}{c}\text { Prevalence of } \\
\text { Avulsions }\end{array}$ \\
\hline C5 & $0(19)$ & 0 \\
C6 & $3(19)$ & 0.16 \\
C7 & $6(19)$ & 0.32 \\
C8 & $6(19)$ & 0.32 \\
T1 & $7(19)$ & 0.37 \\
All levels & $22(95)$ & 0.23 \\
\hline
\end{tabular}

Subtler isolated rootlet findings (ie, findings C, D, and E) did not improve the predictive value of either CT or MR myelography for complete nerve root avulsion. These findings, in the absence of pseudomeningoceles on imaging, may be indicators of proximal nerve insult that cannot be detected on surgical exploration (ie, partial nerve root avulsion). Further study to determine their relevance for surgical planning is necessary. Correlation with proximal nerve stump histopathology and results of nerve grafting may provide further insights.

Chow et $\mathrm{al}^{14}$ previously reported that the additional finding of absent rootlets associated with a pseudomeningocele increased specificity from 0.85 to 0.98 for complete nerve root avulsion. We did not find this difference in our cohort because there were absent rootlets associated with 18 of 19 pseudomeningoceles on CT and with 17 of 17 pseudomeningoceles on MR imaging. The common finding of absent rootlets with pseudomeningoceles in our study may reflect the relatively high prevalence of severe injuries (ie, Narakas 3 and 4) in our cohort compared with that of Chow et al. ${ }^{14}$ We identified avulsions in $23 \%$ of nerve roots examined, whereas Chow et al reported a rate of $14 \%$. Similar to the study of
Steens et $\mathrm{al},{ }^{12}$ the number of patients with at least 1 avulsion was $52 \%$ in our cohort versus $56 \%$ in theirs. Steens et al also found pseudomeningoceles with intact rootlets to be rare, occurring in only $0.5 \%$ of root levels analyzed.

The lack of relevance of subtle nerve root findings for the prediction of complete avulsion, as well as the uncommon finding of pseudomeningoceles with present rootlets, may help explain the similar performance of CT and MR myelography in our study. One of the main advantages of CT myelography over MR imaging-based techniques is the higher spatial resolution that can be achieved in clinically acceptable scanning times. The effective spatial resolution of CT myelography by using the acquisition protocol at our institution was $0.4-0.5 \mathrm{~mm}$ isotropic. For MR myelography, the resolution ranged between 0.5 and $1.0 \mathrm{~mm}$ isotropic by using a fully rewound coherent gradientecho sequence, depending on the required coverage and time constraints.

Because the more conspicuous imaging findings proved to be most predictive of complete nerve avulsion, the weakness of MR imaging in terms of spatial resolution was rendered less significant. In addition, improvements in MR imaging hardware and sequence design have allowed acquisition of progressively higher resolution imaging within acceptable scanning times. We were consistently able to assess the presence or absence of nerve roots in this study by using MR myelography, in contrast to prior studies. ${ }^{43}$ As the impact of subtler nerve root findings on surgical planning and outcomes is elucidated, the relevance of high-resolution nerve root assessment may become clearer. Furthermore, nerve root status may prove more relevant in patient populations with less severe injury grades. For these reasons, continued ad- 
vancement toward the acquisition of high-resolution MR myelographic images remains the ideal.

Confidence ratings were highly correlated between the 2 radiologists and were significantly better on CT myelography because of better spatial resolution compared with MR imaging (Fig 2). We found that MR myelography acquired with voxel sizes of 0.5-0.6 mm was sufficient for high-confidence evaluation commensurate with CT myelography; $0.7-0.8 \mathrm{~mm}$ voxel size yielded intermediate confidence ratings on average, while $\geq 0.9 \mathrm{~mm}$ voxel size led to severely diminished confidence ratings.

Similar to authors of other studies, we report predictive values by using sensitivity, specificity, and predictive values $^{14-16,18,19,35-37}$; and we used clinical examination and/or findings on extradural surgical exploration as our reference standard for the detection of root avulsions. ${ }^{14-16,18,19,35,37,45}$ Hemilaminectomy and opening of the dura mater would provide a more accurate reference standard and have been used to assess CT, ${ }^{13,27} \mathrm{MR}$ imaging, ${ }^{32}$ and both CT and MR imaging ${ }^{46}$ findings in adults. However, the procedure involves significant morbidity and is not performed in infants for neonatal brachial plexus palsy reconstruction.

Given that the decision for surgical treatment at our center is based on clinical examination, our imaging studies were designed to assist with surgical planning only and not to screen for injuries. Our myelography protocols are not optimized to detect more distal extraforaminal neuromas; thus, clinical and imaging findings could not be directly compared.

Accumulation of more CT and MR myelograms to compare diagnostic values would make our conclusions more robust. We had no subjects born breech (in which there is a higher likelihood of C5 and C6 avulsions), and our cohort size did not allow subgroup analysis according to nerve root level. However, this study was initiated as a quality improvement audit following a 3.5-year period during which we performed both CT and MR myelography preoperatively. While both CT and MR myelography are frequently used together and are thought to be complementary, ${ }^{46-48}$ given the findings of this study, we can no longer justify routinely performing both CT and MR myelography in the evaluation of neonatal brachial plexus palsy at our institution.

\section{CONCLUSIONS}

The predictive values of CT and MR myelography are similar for the detection of complete nerve root avulsion in neonatal brachial plexus palsy, and we found no benefit to the combined use of CT and MR imaging over MR myelography alone. Although radiologists' confidence ratings were significantly better with CT myelography, findings on CT and MR myelography were highly correlated. Given the advantages of MR myelography, it is now the single technique for preoperative evaluation of nerve root avulsion at our institution.

\section{ACKNOWLEDGMENTS}

The authors thank Kathryn Whitlock, Center for Clinical and Transitional Research, Seattle Children's Hospital, for her guidance and assistance with statistical analysis.

\section{REFERENCES}

1. Pondaag W, Malessy MJ, van Dijk JG, et al. Natural history of obstetric brachial plexus palsy: a systematic review. Dev Med Child Neurol 2004;46:138-44

2. Hale HB, Bae DS, Waters PM. Current concepts in the management of brachial plexus birth palsy. J Hand Surg 2010;35A:322-31

3. Stevens JH. Brachial plexus paralysis: By JH. Stevens, M.D., 1934. Clin Orthop Relat Res 1988;237:4-8

4. Benjamin K. Part 1. Injuries to the brachial plexus: mechanisms of injury and identification of risk factors. Adv Neonatal Care 2005;5:181-89

5. Narakas AO. Obstetrical brachial plexus injuries. In: Lamb DW, ed. The Paralyzed Hand. Edinburgh, Scotland: Churchill Livingstone; 1987:116-35

6. Buchanan EP, Richardson R, Tse R. Isolated lower brachial plexus (Klumpke) palsy with compound arm presentation: case report. J Hand Surg Am 2013;38:1567-70

7. al-Qattan MM, Clarke HM, Curtis CG. Klumpke's birth palsy: does it really exist? J Hand Surg $\mathrm{Br}$ 1995;20:19-23

8. Hentz VR. Congenital brachial plexus exploration. Tech Hand Up Extrem Surg 2004;8:58-69

9. Borschel GH, Clarke HM. Obstetrical brachial plexus palsy. Plast Reconstr Surg 2009;124(suppl):144e-55e

10. Waters PM. Comparison of the natural history, the outcome of microsurgical repair, and the outcome of operative reconstruction in brachial plexus birth palsy. J Bone Joint Surg Am 1999;81:649-59

11. Malessy MJ, Pondaag W. Obstetric brachial plexus injuries. Neurosurg Clin of N Am 2009;20:1-14

12. Steens SC, Pondaag W, Malessy MJ, et al. Obstetric brachial plexus lesions: CT myelography. Radiology 2011;259:508-15

13. Carvalho GA, Nikkhah G, Matthies C, et al. Diagnosis of root avulsions in traumatic brachial plexus injuries: value of computerized tomography myelography and magnetic resonance imaging. J Neurosurg 1997;86:69-76

14. Chow BC, Blaser S, Clarke HM. Predictive value of computed tomographic myelography in obstetrical brachial plexus palsy. Plast Reconstr Surg 2000;106:971-77

15. Vanderhave KL, Bovid K, Alpert H, et al. Utility of electrodiagnostic testing and computed tomography myelography in the preoperative evaluation of neonatal brachial plexus palsy. J Neurosurg Pediatr 2012;9:283-89

16. Terzis JK, Novikov ML. Radiological and electrophysiological detection of nerve roots avulsion in patients with birth-related brachial plexus paralysis. Semin Plast Surg 2005;19:24

17. Van Ouwerkerk W. Preoperative investigations in obstetric brachial plexus palsy. Semin Plast Surg 2005;19:17

18. Bertelli JA, Ghizoni MF. Use of clinical signs and computed tomography myelography findings in detecting and excluding nerve root avulsion in complete brachial plexus palsy. J Neurosurg 2006;105: 835-42

19. Yamazaki H, Doi K, Hattori Y, et al. Computerized tomography myelography with coronal and oblique coronal view for diagnosis of nerve root avulsion in brachial plexus injury. J Brachial Plex Peripher Nerve Inj 2007;2:16

20. Klein KM, Shiratori K, Knake S, et al. Status epilepticus and seizures induced by iopamidol myelography. Seizure 2004;13:196-99

21. Gelfand MS, Abolnik IZ. Streptococcal meningitis complicating diagnostic myelography: three cases and review. Clin Infect Dis 1995;20:582-87

22. Chitnis AS, Guh AY, Benowitz I, et al. Outbreak of bacterial meningitis among patients undergoing myelography at an outpatient radiology clinic. J Am Coll Radiol 2012;9:185-90

23. Pearce MS, Salotti JA, Little MP, et al. Radiation exposure from CT scans in childhood and subsequent risk of leukaemia and brain tumours: a retrospective cohort study. Lancet 2012;380:499-505

24. Mathews JD, Forsythe AV, Brady Z, et al. Cancer risk in 680,000 people exposed to computed tomography scans in childhood or 
adolescence: data linkage study of 11 million Australians. $B M J$ 2013;346:f2360

25. van Ouwerkerk WJ, Strijers RL, Barkhof F, et al. Detection of root avulsion in the dominant $\mathrm{C} 7$ obstetric brachial plexus lesion: experience with three-dimensional constructive interference in steadystate magnetic resonance imaging and electrophysiology. Neurosurgery 2005;57:930-40

26. Yoshikawa T, Hayashi N, Yamamoto S, et al. Brachial plexus injury: clinical manifestations, conventional imaging findings, and the latest imaging techniques. Radiographics 2006;26(suppl 1):S133-43

27. Oberle J, Antoniadis G, Rath SA, et al. Radiological investigations and intra-operative evoked potentials for the diagnosis of nerve root avulsion: evaluation of both modalities by intradural root inspection. Acta Neurochir (Wien) 1998;140:527-31

28. O'Shea K, Feinberg JH, Wolfe SW. Imaging and electrodiagnostic work-up of acute adult brachial plexus injuries. J Hand Surg Eur Vol 2011;36:747-59

29. Terzis JK, Kostas I. Outcomes with suprascapular nerve reconstruction in obstetrical brachial plexus patients. Plast Reconstr Surg 2008; $121: 1267-78$

30. Vargas MI, Viallon M, Nguyen $D$, New approaches in imaging of the brachial plexus. Eur J Radiol 2010;74:403-10

31. Amrami KK, Port JD. Imaging the brachial plexus. Hand Clin 2005;21:25-37

32. Tsai PY, Chuang TY, Cheng H, et al. Concordance and discrepancy between electrodiagnosis and magnetic resonance imaging in cervical root avulsion injuries. J Neurotrauma 2006;23:1274-81

33. Ochi M, Ikuta $Y$, Watanabe $M$, et al. The diagnostic value of MRI in traumatic brachial plexus injury. J Hand Surg Br 1994;19:55-59

34. Hems TE, Birch R, Carlstedt T. The role of magnetic resonance imaging in the management of traction injuries to the adult brachial plexus. J Hand Surg Br 1999;24:550-55

35. Abul-Kasim K, Backman C, Björkman A, et al. Advanced radiological work-up as an adjunct to decision in early reconstructive surgery in brachial plexus injuries. J Brachial Plex Peripher Nerve Inj 2010;5:14

36. Gasparotti R, Ferraresi S, Pinelli L, et al. et al. Three-dimensional MR myelography of traumatic injuries of the brachial plexus. AJNR Am J Neuroradiol 1997;18:1733-42
37. Doi K, Otsuka K, Okamoto Y, et al. Cervical nerve root avulsion in brachial plexus injuries: magnetic resonance imaging classification and comparison with myelography and computerized tomography myelography. J Neurosurg 2002;96:277-84

38. Nakamura T, Yabe Y, Horiuchi Y, et al. Magnetic resonance myelography in brachial plexus injury. J Bone Joint Surg $\mathrm{Br}$ 1997;79:764-69

39. Smith AB, Gupta N, Strober J, et al. Magnetic resonance neurography in children with birth-related brachial plexus injury. Pediatr Radiol 2007;38:159-63

40. Francel PC, Koby M, Park TS, et al. et al. Fast spin-echo magnetic resonance imaging for radiological assessment of neonatal brachial plexus injury. J Neurosurg 1995;83:461-66

41. Yilmaz K, Calişkan M, Oge E, et al. Clinical assessment, MRI, and EMG in congenital brachial plexus palsy. Pediatr Neurol 1999;21: $705-10$

42. Miller SF, Glasier CM, Griebel ML, et al. Brachial plexopathy in infants after traumatic delivery: evaluation with MR imaging. Radiology 1993;189:481-84

43. Medina LS, Yaylali I, Zurakowski D, et al. Diagnostic performance of MRI and MR myelography in infants with a brachial plexus birth injury. Pediatr Radiol 2006;36:1295-99

44. Gosk J, Hendrich B, Wiacek R, et al. Assessment of the usefulness of $\mathrm{X}$-ray myelography and magnetic resonance myelography, performed with an open low-field device, in diagnosing perinatal preganglionic injuries of the brachial plexus. Arch Med Sci 2012;8:678-83

45. Malessy MJ, Pondaag W, van Dijk JG. Electromyography, nerve action potential, and compound motor action potentials in obstetric brachial plexus lesions: validation in the absence of a "gold standard." Neurosurgery 2009;65(4 suppl):A153-59

46. Penkert G, Carvalho GA, Nikkhah G, et al. Diagnosis and surgery of brachial plexus injuries. J Reconstr Microsurg 1999;15:3-8

47. Bowen BC, Seidenwurm DJ, for the Expert Panel on Neurologic Imaging. Plexopathy. AJNR Am J Neuroradiol 2008;29:400-02

48. Belzberg AJ, Dorsi MJ, Storm PB, et al. Surgical repair of brachial plexus injury: a multinational survey of experienced peripheral nerve surgeons. J Neurosurg 2004;101:365-76 